\title{
Wide Sets, ZFCU, and the Iterative Conception*
}

\author{
Christopher Menzel \\ Forthcoming in Journal of Philosophy
}

\section{Introduction}

In the "intended" models of (first-order) ZFC - Zermelo-Fraenkel set theory with the axiom of Choice - there are only sets. ZFCU is ZFC modified to allow for the existence of urelements, or atoms, i.e., things that can be members of sets but are not themselves sets and do not themselves have members. Consider, then, the following consequence of David Lewis's (1986) unqualified principle of Recombination:

R For any cardinal number $\kappa$, it is possible that there are at least $\kappa$ atoms. ${ }^{1}$

Daniel Nolan (1996) has shown that, in the context of Lewis's modal realism, $\mathbf{R}$ entails:

$$
\mathbf{A}_{\infty} \text { For any cardinal number } \mathcal{\kappa} \text {, there are at least } \mathcal{\kappa} \text { atoms. }{ }^{2}
$$

${ }^{*}$ My sincere thanks to Albert Anglberger, Roy Cook, Thomas Forster, Jeffrey Ketland, Hannes Leitgeb, and Godehard Link for their insightful questions comments on earlier versions of, and talks based on, this paper. I am especially grateful to Prof. Dr. Leitgeb for graciously hosting me for the 2011-12 academic year at the Munich Center for Mathematical Philosophy. I cannot imagine a more congenial and exciting environment in which to think and work. My thanks also to the Humboldt Stiftung for its support of the MCMP.

${ }^{1}$ Lewis's actual principle $(1986,88-89)$ is that, for any $\kappa$ and any world $W$, it is possible that there exist $\kappa$ "duplicates" of the objects that exist in $W$. This obviously entails $\mathbf{R}$.

${ }^{2}$ More recently, Ted Sider (2009) has shown that basically the same argument can be reconstructed in the context of Timothy Williamson's (2002) theory of necessary existence. 
Although there are many deep and interesting issues in the metaphysics of modality to explore here, my central concern in this paper is with an apparent disconnect that emerges in the conceptual foundations of set theory. Specifically, let SoA be the proposition

SoA There is a set of all atoms

and let So $_{\infty}$ be the conjunction SoA $\wedge \mathbf{A}_{\infty}$ asserting that the set of atoms exists and is larger than any cardinal number. It is very easy to show that ZFCU entails $\sim \mathbf{S o A}_{\infty}$, i.e., that no such set exists (see §1.3). However, as Lewis himself has argued (1986, 104ff), our best and most intuitive conception of sets - the so-called iterative conception - seems to be entirely consistent with So $_{\infty}$. But this is a bit puzzling, as the iterative conception is typically considered to provide the motivation for the intended models of ZFCU, viz., various limit stages of the so-called cumulative hierarchy. ${ }^{3}$ So whence the disconnect?

In this paper I diagnose the source of the disconnect and investigate possibilities for modifying ZFCU so as to render it consistent with $\mathbf{S o A}_{\infty}$ without undermining its intuitive moorings in the iterative conception. Those familiar with some of the issues here will not be surprised to find that I focus on the schema F of Replacement. As is well known, F expresses a certain "limitation of size" assumption about sets. This assumption is critical to the derivation of $\sim \mathbf{S o} \mathbf{A}_{\infty}$ in ZCFU and I will argue that it is the source of the disconnect in question. In response, I first propose restricting $\mathbf{F}$ so that it applies only to "small" sets, which divests it of its power to limit set size. This restriction blocks the derivation of $\sim \mathbf{S o A}_{\infty}$ but, due to the presence of the Powerset axiom PS, leaves us with a new disconnect between size and number. I argue, however, that Cantor's notion of the absolute infinite provides us with grounds for a principled restriction on PS that avoids this disconnect and still preserves a robust, though qualified, iterative conception of set. Finally, the loss of certain desirable theorems due to the restriction on PS leads me to strengthen the modified Replacement schema so that it can applied to large sets - paradox is avoided by incorporating what might be called a limitation of height assumption rather than a limitation of size.

\footnotetext{
${ }^{3}$ As first described in detail by Zermelo (1930) himself. This paper is translated, with an excellent introduction by Kanamori, in Zermelo 2010, 392-431.
} 
In an appendix, it is shown that the suggested modifications are provably consistent with SoA $_{\infty}$ (relative to an extension of ZFC).

\section{ZFCU}

We begin by making the axioms of ZFCU explicit and laying out some important definitions and theorems. Our language includes the membership predicate $\epsilon$ along with a unary predicate Set. A separate predicate for atoms is of course unnecessary, as an atom in the context of ZFCU is by definition anything that is not a set. The thesis SoA can thus be formalized in the obvious way:

SoA $\exists x \forall y(y \in x \leftrightarrow \sim \operatorname{Set}(y))$.

In addition to the usual apparatus of first-order logic with identity we will also avail ourselves of the quantifier $\exists ! v \varphi$ (defined as usual) signifying that exactly one thing satisfies $\varphi$. In "impure" set theories like ZFCU, propositions about sets alone are often more conveniently expressed in terms of dedicated variables $A, B, C, \ldots$ that range only over sets; we will follow this practice here. ${ }^{4}$ Finally, for variables $v$ and terms $\tau$, we will often abbreviate sentences of the form $\forall v(v \in \tau \rightarrow \varphi)$ and $\exists v(v \in \tau \wedge \varphi)$ as $\forall v \in \tau \varphi$ and $\exists v \in \tau \varphi$, respectively.

\subsection{The Axioms}

Our first two axioms characterize the most fundamental properties of our two primitive predicates:

Set $y \in x \rightarrow \operatorname{Set}(x)$

Ext $\forall x(x \in A \leftrightarrow x \in B) \rightarrow A=B$.

The axioms of Pairing and Union and the schema of Separation are perhaps the most fundamental principles for "generating" new sets from given sets and atoms:

\footnotetext{
${ }^{4} \mathrm{~A}$ bit more exactly, we will often write $\exists A \varphi_{A}^{x}$ and $\forall A \varphi_{A}^{x}$ for $\exists x(\operatorname{Set}(x) \wedge \varphi)$ and $\forall x(\operatorname{Set}(x) \rightarrow \varphi$ ), respectively (where a variable other than ' $A$ ' is chosen as needed to avoid untoward collisions). These can be thought of as either notational abbreviations or as theorems in a conservative extension of ZFCU as desired.
} 
$\operatorname{Pg} \exists A \forall z(z \in A \leftrightarrow(z=x \vee z=y))$

Un $\exists B \forall x(x \in B \leftrightarrow \exists C \in A x \in C)$

Sep $\exists B \forall x(x \in B \leftrightarrow(x \in A \wedge \varphi))$, where ' $B$ ' does not occur free in $\varphi$.

Given Ext, Pg, Un, and Sep, we can justify the usual definitions of $\varnothing$, the general union and intersection operators $U$ and $\bigcap$, and their binary counterparts $U$ and $\cap$. We will also avail ourselves of set abstracts $\{x: \varphi\}$ when we can prove that the objects satisfying $\varphi$ form a set, and we let $\left\{a_{1}, \ldots, a_{n}\right\}$ abbreviate $\left\{x: x=a_{1} \vee \ldots \vee x=a_{n}\right\}$ (which exists by Pg and Un). We define the subset relation as usual, except that we explicitly restrict it to sets:

$$
x \subseteq y={ }_{d f} \operatorname{Set}(x) \wedge \operatorname{Set}(y) \wedge \forall z(z \in x \rightarrow z \in y) .
$$

These definitions and conventions are useful for simplifying the remaining axioms.

Fnd $A \neq \varnothing \rightarrow \exists x \in A x \cap A=\varnothing$

Inf $\exists A(\varnothing \in A \wedge \forall x(x \in A \rightarrow x \cup\{x\} \in A))$

PS $\exists B \forall x(x \in B \leftrightarrow x \subseteq A)$.

By PS and Ext, every set $A$ has a unique power set, so let $\wp(A)=_{d f}\{x: x \subseteq A\}$.

The above axioms constitute what is often called $Z$ set theory with urelements, $Z U$, as, with the exception of Fnd, versions of them are all included in Zermelo 1908, the first reasonably rigorous crack at what was to become ZFC. Zermelo came to embrace Fnd as well as it became clearer to him that the iterative conception underlay the intended models of his axioms and it is included explicitly in the axiomatization he adopted in his 1930 analysis of the those models. ${ }^{5}$

Also included in Zermelo's 1908 axiomatization was the axiom of Choice. As the issues at hand are rendered all the more difficult if there are nonwell-orderable sets, we will avail ourselves freely of the axiom. (I am also among those who, perhaps quaintly, consider the axiom to be true.) To simplify its expression, let us say that $x$ is choice-friendly, $C F(x)$, if $x$ is a set of nonempty pairwise disjoints sets:

\footnotetext{
${ }^{5}$ See Moore 1978, 1980 and Kanimori's introduction to Zermelo 1908 in Zermelo 2010, 390-399.
} 


$$
C F(x) \equiv_{d f} \operatorname{Set}(x) \wedge \forall y \in x(y \neq \varnothing \wedge \forall z \in x(z \neq y \rightarrow y \cap z=\varnothing)) .
$$

Choice then says simply that every choice-friendly set $x$ has a choice set, a set containing exactly one member of each member of $x$ :

AC $C F(x) \rightarrow \exists C \forall B \in x \exists ! z \in B z \in C$.

Finally, critical to set theory's power - and to matters here - is the axiom schema of Replacement, added by Fraenkel, which tells us, roughly, that the values $y$ of any definable functional mapping $\psi(x, y)$ on a set $A$ jointly constitute a set:

F $\forall x \in A \exists ! y \psi \rightarrow \exists B \forall y(y \in B \leftrightarrow \exists x \in A \psi)$, where ' $B$ ' does not occur free in $\psi$.

By ZFCU, then, we mean all of the above axioms.

\subsection{Ordinals, Cardinals, and Cantor's Theorem}

In the universe of ZFC, all sets are "pure", unsullied by the presence of atoms anywhere in their membership ancestry. It will be important for a number of purposes to characterize this notion formally within ZFCU. First, we say that an object $x$ is transitive just in case it is a set such that all of its set members are subsets:

$$
\operatorname{Tran}(x) \equiv_{d f} \operatorname{Set}(x) \wedge \forall A \in x A \subseteq x .
$$

Define $y$ to be a transitive closure of $x$ just in case $y$ is a smallest transitive superset of $x$ :

$$
T C(x, y) \equiv_{d f} x \subseteq y \wedge \operatorname{Tran}(y) \wedge \forall z((\operatorname{Tran}(z) \wedge x \subseteq z) \rightarrow y \subseteq z) .
$$

Thus, if $B$ is a transitive closure of $A$, then $B$ contains the members of $A$, the members of those members, and so on; $B$, that is, contains all of $A$ 's ancestors vis-á-vis membership. Given this, we can say that a set is pure if it has a transitive closure that contains only sets:

$$
\operatorname{Pure}(x) \equiv_{d f} \exists y(T C(x, y) \wedge \forall z \in y \operatorname{Set}(z)) .^{6}
$$

\footnotetext{
${ }^{6}$ In fact, in ZFCU, every set has a unique transitive closure and, hence, we could have provided a more elegant definition of Pure in terms of a transitive closure function; but see fn 19 below.
} 
Given the above, we define the ordinal numbers to be pure sets that are transitive and totally ordered (hence, by Fnd, well-ordered) by membership:

$$
\operatorname{Ord}(x) \equiv_{d f} \operatorname{Pure}(x) \wedge \operatorname{Tran}(x) \wedge \forall y z \in x(y \in z \vee z \in y \vee y=z) .^{7}
$$

The less than relation on ordinals is then understood as usual simply as membership:

$$
x<y \equiv_{d f} \operatorname{Ord}(x) \wedge \operatorname{Ord}(y) \wedge x \in y .
$$

The lower case Greek letters $\alpha, \beta$, and $\lambda$ will be reserved as variables ranging over ordinals.

By Sep and Ext there is a unique smallest set $\omega$ satisfying Inf and it is easy to show that $\omega$ is an ordinal; we identify the members of $\omega$ with the natural numbers. By Fnd, every condition $\varphi(x)$ satisfied by any ordinals is satisfied by a (unique) least ordinal. Thus, in particular, every set $B$ of ordinals has a least element $\inf B$, a least upper bound $\sup B$, and a least strict upper bound $\sup ^{+} B .^{8}$

Functions are understood as usual as sets of ordered pairs, where an ordered pair $\langle a, b\rangle=d f\{\{a\},\{a, b\}\}$ is defined á la Kurotowski (and, hence, exists by $\operatorname{Pg}$ given $a$ and $b$ ). Say that $A$ is as large as $B$ or, alternatively, that $A$ is the same size as $B, A \approx B$, if there is an bijection from $A$ onto $B$; that $A$ is no larger than $B$ or, alternatively, that $B$ is at least as large as $A, A \leqslant B$, if there is an injection of $A$ into $B$; and that $A$ is smaller than $B$ or that $B$ is larger than $A, A<B$, if $A \leqslant B$ but not $A \approx B$. A set $A$ is finite if $A$ is as the same size as a natural numbers, i.e., an ordinal $\alpha \in \omega$.

It is a well-known theorem of ZFCU that every set is the same size as some ordinal:

\section{(1) $\forall A \exists \alpha A \approx \alpha$.}

Proof (ZFCU). Let $A$ be a set. By AC and PS there is a well-ordering $R$ of $A$. For $a \in A$, let $a_{R}$ be the set of $R$-predecessors of $a$, i.e., $a_{R}=\{c \in A: R c a\}$. By Sep, let $C$ consist of those $a \in A$ such that $a_{R}$ is isomorphic to some

\footnotetext{
${ }^{7}$ The restriction to pure sets prevents impure "ordinals" built up from a single urelement $a-$ e.g., $\{a,\{a\},\{a,\{a\}\}\}-$ from counting as genuine ordinals.

${ }^{8}$ These are of course the least ordinals satisfying, respectively, the conditions $x \in B$, $\forall \alpha \in B \alpha \leq x$, and $\forall \alpha \in B \alpha<x$.
} 
(unique) ordinal $\beta$, i.e., $C=\left\{a \in A: \exists \beta a_{R} \simeq \beta\right\} .{ }^{9}$ Since the mapping $a_{R} \simeq \beta$ is functional, by $\mathbf{F}$, the set $B=\left\{\beta: \exists a \in C a_{R} \simeq \beta\right\}$ exists. By PS and Sep we have the function $\left.f=\left\{\langle a, \beta\rangle \in C \times B: a_{R} \simeq \beta\right\}\right)$. It is easy to verify that $B$ is an ordinal, that $f$ is a one-to-one correspondence from $C$ to $B$, and that $A=C$.

It is important for purposes here to note that the use of the Replacement schema $\mathbf{F}$ in this proof is essential. ${ }^{10}$

It follows from (1) that there is always a least ordinal as large as $A$, which we identify with the cardinality of $A$ :

$$
|A|=_{d f} \inf \{\alpha: A \approx \alpha\} .
$$

A cardinal number generally is thus defined to be any such ordinal:

$$
\operatorname{Card}(x) \equiv_{d f} \exists A x=|A|^{11}
$$

I will reserve the Greek letters $\kappa$ and $\mu$ as variables ranging over cardinals.

It is a simple consequence of the definition of cardinality that differences in relative size correspond exactly to differences in cardinality:

$$
A \prec B \leftrightarrow|A|<|B| .
$$

What gives this proposition bite, of course, is Cantor's famous theorem, which tells us that differences in size extend into the infinite:

(3) $A \prec \wp(A)$.

Proof $(Z U)$. The function $f(x)=\{x\}$ from $A$ into $\wp(A)$ is obviously an injection, so $A \leqslant \wp(A)$. However, for any function $g$ from $A$ into $\wp(A)$, the diagonal set $\{x \in A: x \notin g(x)\}$ for $g$ cannot be in the range of $g$, on pain of contradiction. Hence, $g$ cannot be a bijection, i.e., $A \approx B$.

It follows straightaway from (2), (3) and the definition of cardinality that no set's cardinality is maximal:

$$
\forall A \exists \kappa|A|<\kappa \text {. }
$$

\footnotetext{
${ }^{9} \mathrm{By} a_{R} \simeq \beta$, of course, we mean $\left\langle a_{R}, R\lceil a\rangle \simeq\langle\beta, \in \uparrow \beta\rangle\right.$.

${ }^{10}$ We know this because, e.g., level $V_{\omega+\omega}$ of the cumulative hierarchy (Devlin 1991, §2.2) is a model of ZU+AC that contains uncountable sets (e.g., $\wp(\omega) \in V_{\omega+2}$ ) but only countable ordinals $\alpha<\omega+\omega$.

${ }^{11}$ It is easy to show that this definition of the cardinals is equivalent in ZFCU to another common definition that takes the cardinals to be initial ordinals: $\operatorname{Card}(x) \leftrightarrow(\operatorname{Ord}(x) \wedge$ $\forall \beta \in x \beta<x)$.
} 


\subsection{ZFCU $\sim \sim$ SoA $_{\infty}$}

In set theory, to say there are at least $\kappa$ atoms is to say that there is a function $f$ mapping each $\alpha<\mathcal{K}$ uniquely to an atom. Given such a function $f$, by Un and Sep, the set $\{a \in \bigcup \bigcup f: \exists \alpha<\kappa f(\alpha)=a\}$ of all the atoms in question exists. Hence, thesis $\mathbf{A}_{\infty}$ is just as well expressed by saying simply that, for every $\mathcal{K}$, there is a set of atoms at least as big as $\kappa$ :

$$
\mathbf{A}_{\infty} \forall \kappa \exists A(\forall x \in A \sim \operatorname{Set}(x) \wedge \kappa \leqslant A) .
$$

Given SoA and $\mathbf{A}_{\infty}$ as rendered above, it is easy to prove $\sim \operatorname{So} \mathbf{A}_{\infty}$ in ZFCU. For assume $\mathbf{A}_{\infty}$ and let $B$ be any set of atoms. By (4), there is a cardinal $\mathcal{K}$ such that $|B|<\kappa$ and so by (2) $B<\kappa$. By $\mathbf{A}_{\infty}$ there is a set $A$ of atoms of at least size $\kappa$, i.e., such that $\kappa \leqslant A$. It follows directly that $B$ is smaller than $A$, $B<A$, and, hence, that $B$ cannot be the set of all atoms. But $B$ was chosen arbitrarily, so $\sim$ SoA. By conditional proof, we conclude $\mathbf{A}_{\infty} \rightarrow \sim$ SoA, which is just $\sim$ SoA $_{\infty} \cdot{ }^{12}$

\section{The Iterative Conception and SoA $_{\infty}$}

Let us now consider the argument that the iterative conception is consistent with $\mathbf{S o A}_{\infty}$. We argue first that the iterative conception entails the weaker conjunct SoA. The key intuitive idea in the iterative conception is that sets are "formed" in a series of cumulative "stages" from an initial stock of atoms. The first stage consists of all the sets - including the empty set - that can be formed from the atoms; subsequent stages (including, we may assume, "limit" stages that have no immediate predecessor) consist of all the sets that can be formed from the atoms and the sets formed in preceding stages. ${ }^{13}$

\footnotetext{
${ }^{12}$ Choice is in fact not essential to the theorem: Assume $\operatorname{SoA}_{\infty}$, i.e., $\operatorname{SoA} \wedge \mathbf{A}_{\infty}$. By SoA, let $A^{*}$ be the set of all atoms. By $\mathbf{A}_{\infty}$, every cardinal can be injected into some subset $B$ of $A^{*}$ and, hence, into $A^{*}$ itself. It follows in ZFU that every well-orderable set can be injected into $A^{*}$. But that directly contradicts Hartogs' (1915) Theorem (which is provable in ZU). So $\sim$ SoA $_{\infty}$. (My thanks to Thomas Forster for pointing out to me that the proof of Hartogs' Theorem does not require Replacement.)

${ }^{13}$ This description of the hierarchy of stages — which follows Boolos 1971 - thus doesn't square exactly with the cumulative hierarchy of Zermelo 1930 and, for that matter, the accounts in most modern textbooks like Devlin 1991, where limit stages simply accumulate the members of the preceding stages.
} 
The "process" of set formation thus yields an ever-expanding hierarchy of stages built up over the atoms as depicted in Figure 1, with an inner cone of pure sets built up from the empty set alone. The bottom "disk" thus represents the first stage containing all sets of atoms; "disks" occurring higher up represent the cumulative stages arising subsequently in the process. The sets themselves, therefore, are exactly those collections that are formed at some stage. The argument for SoA, then, is simply this: One of the sets that can be formed from the atoms is the set of all of them. They are, after all, all there to be collected at the very beginning of the process. Hence, the set of all atoms is formed at the very first stage.

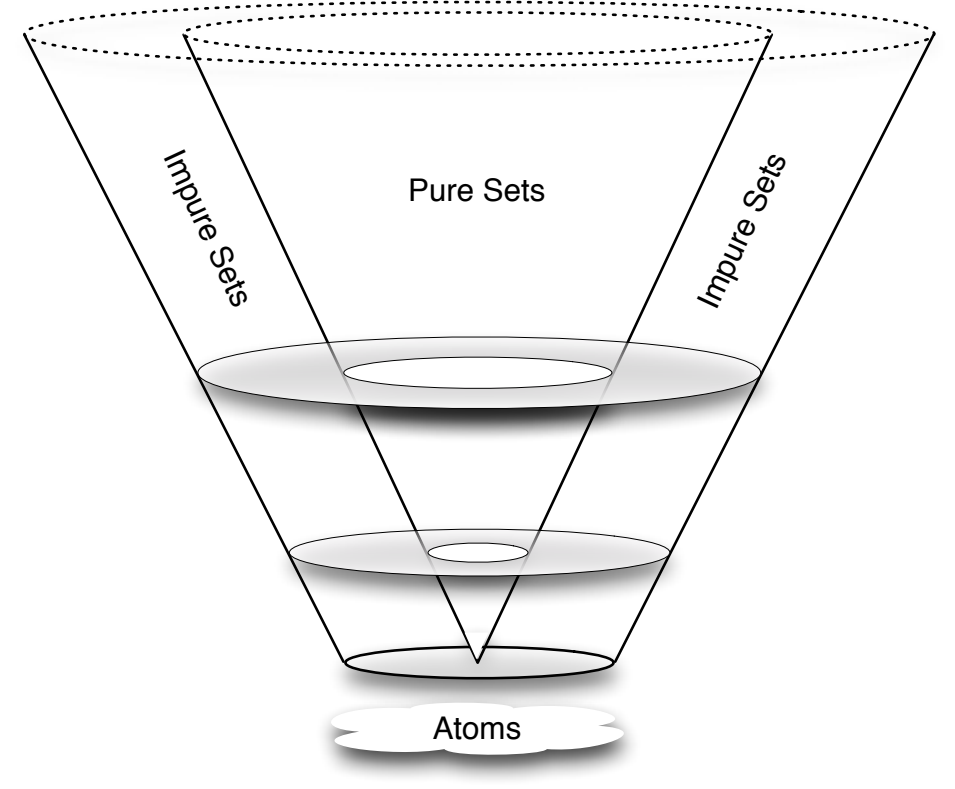

Figure 1: The cumulative hierarchy of stages

The argument can be more rigorously expressed in terms of the notion of rank, defined recursively as follows:

Rnk $\operatorname{rnk}(x)=\sup ^{+}\{\operatorname{rnk}(y): y \in x\}$.

So the rank of an object with no members - i.e., an atom or the empty set - is 0 ; and that of any set $A$ in general is the least ordinal greater than the ranks of $A$ 's members. Thus, the rank of a set $A$ corresponds directly to the stage at which it can only first be formed, the first stage of the process such 
that all of $A$ 's members have been formed in prior stages. ${ }^{14}$ The informal idea that each set is formed from atoms and sets that first occur "earlier" in the process of set formation is therefore captured formally in the fact that the rank of any member of a set $A$ will always be less than $A$ 's rank. The sets, then - as opposed to "proper classes" (like the class On of all ordinals) consisting of objects that are formed at arbitrarily high stages - are exactly those collections that have a rank. ${ }^{15}$ The argument for SoA can now be recast as follows: Atoms have a rank of 0 and, hence, jointly constitute a collection of rank 1 and, hence, a set.

It is easy now to see that $\mathbf{A}_{\infty}$, hence, $\operatorname{So} \mathbf{A}_{\infty}$, is consistent with the iterative conception. The critical point is simply this: Sethood on the iterative conception does not have anything to do with size but with structure: All that matters in regard to the question of whether some things - the atoms, in particular - form a set is whether or not there is an upper bound to their ranks; how many of them there are, even if there are too many to be numbered by any given cardinal, is utterly irrelevant. Thus Lewis $(1986,104)$ : “

We do say that according to the iterative conception of sets, some classes are 'too big to be sets', but this is loose talk. Sheer size is not what matters; rather, the obstacle to sethood is that the members of a [proper] class are not all present at any rank of the iterative hierarchy. But all the individuals, no matter how many there may be, get in already at the ground floor. So, after all, we have no notion what could stop any class of individuals ... from comprising a set.

As we might put it, then, the iterative conception prevents proper classes from counting as sets, not because they are "too big", but because they are "too high". Sets have to be formed at some stage; the overly-large character

\footnotetext{
${ }^{14}$ If we think of the atoms as jointly constituting a sort of "pre-stage" $S_{0}$, then the finite ranks of pure sets are a bit out of sync with the intuitive ordering of stages. For impure sets $A, r n k(A)=\alpha$ iff $A$ is formed at stage $\alpha$, for all $\alpha$. This is true for pure sets $B$ only when $\alpha \geq \omega$ : Assuming $\varnothing$ is first "formed" at the first stage $S_{1}$ with all other sets of atoms, then, for finite $n, B$ is formed at stage $S_{n+1}$ iff $\operatorname{rnk}(B)=n$.

${ }^{15} \mathrm{As}$ is well known, $\mathrm{ZFC}(\mathrm{U})$ does not countenance the existence of proper classes, although there are related set theories notably, von Neumann-Bernays-Gödel set theory, NBG that do; see, e.g., Mendelson 1997, ch. 4.
} 


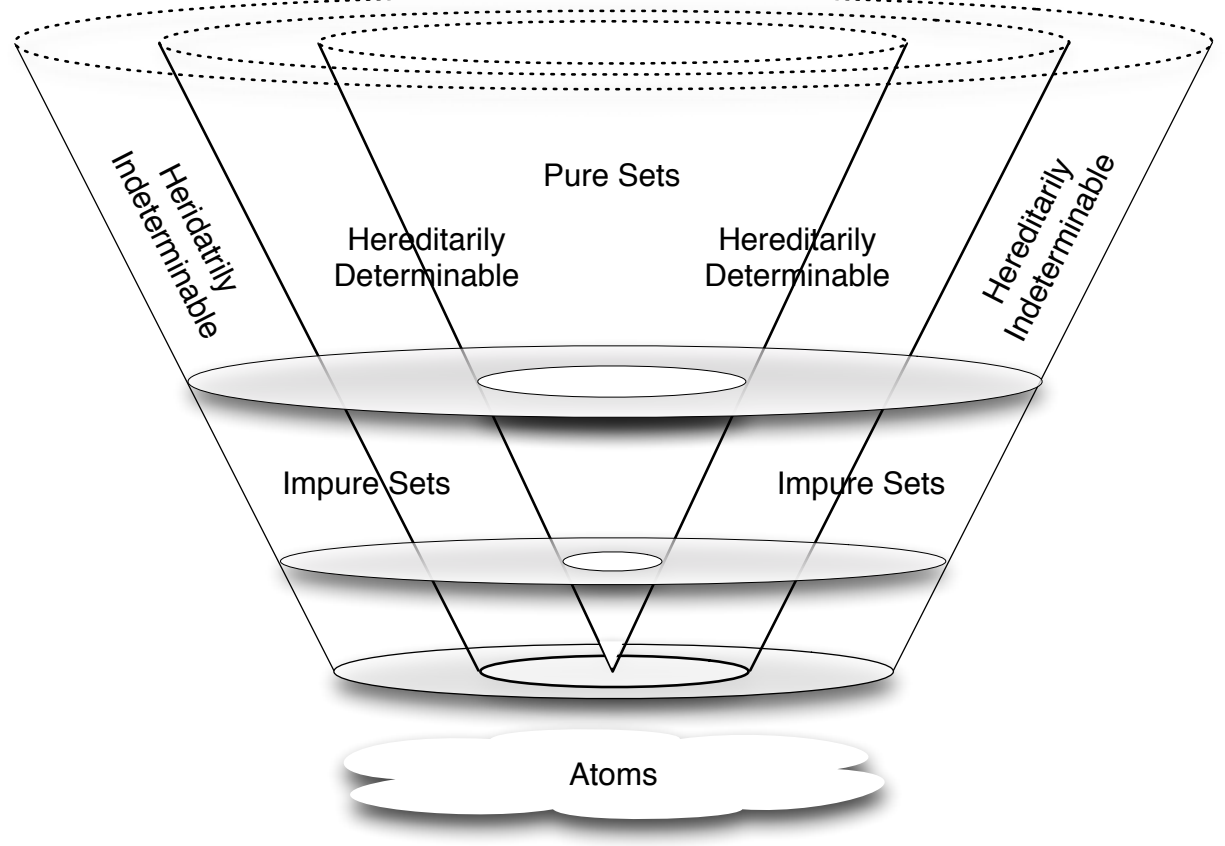

Figure 2: The cumulative hierarchy with wide sets

of proper classes is, at it were, simply a side-effect of this structural flaw. The flaw is not present in the collection of all atoms, even if it is too big — or better, "too wide" - to have a definite cardinality. Hence, there is no reason to deny that it is a set; $\mathbf{S o A}_{\infty}$, that is, is intuitively consistent with the iterative conception.

It therefore seems entirely possible that the universe of sets needs to be conceptualized rather differently than the universe of ZFCU depicted in Figure 1. The simplest revision seems to be one where we just broaden the "disk" at the base of hierarchy to accommodate wide sets, as depicted in Figure $2 .{ }^{16}$ As is clearly seen there, whereas the only interesting division in the universe of ZFCU sets is between the pure and the impure, if $\mathbf{S o A}_{\infty}$ is true, the rather more interesting divide is between the hereditarily determinable and the hereditarily indeterminable - that is, between sets whose transitive closures are narrow and sets whose transitive closures are wide (i.e., sets that are either themselves wide or have wide sets in their ancestry).

\footnotetext{
${ }^{16} \mathrm{~A}$ more conservative option, of course, is simply to admit $\mathbf{A}_{\infty}$ and broaden the base
} 


\section{Making Room for Wide Sets}

\subsection{The Source of the Disconnect}

So there is a disconnect between ZFCU and the iterative conception with regard to the possibility of wide sets. The primary source of the disconnect is the Replacement schema $\mathbf{F}$. The controversies surrounding $\mathbf{F}$ typically revolve around its power to extend the cumulative hierarchy beyond what seems warranted by the iterative conception alone, with its modest stepwise process of set formation, even granting the existence of limit stages. ${ }^{17}$ For, given a set $A$ of any size, $\mathbf{F}$ guarantees that any conceivable way of mapping $A$ "upward" in the hierarchy, no matter how large the "jumps" in the mapping, will always terminate by some stage; hence, the range of the mapping will constitute a set in the very next stage. Equivalently put, the range of such a mapping on $A$ will always be bounded in rank. For example, given the mapping $x \in \omega \wedge y=\aleph_{x}$ - i.e., the mapping $n \mapsto \aleph_{n}$ that takes each natural number $n$ to the $n^{\text {th }}$ transfinite cardinal number $-\mathbf{F}$ guarantees the existence of the limit cardinal $\aleph_{\omega}$ (and thence also a stage containing it). However, F's power is a double-edged sword; and it is the other edge that lies at the heart of the disconnect between $\mathbf{F}$ and the iterative conception that arises vis-á-vis wide sets. For by guaranteeing that the range of any mapping on any set is bounded in rank, $\mathbf{F}$ simultaneously restricts the universe to sets whose width does not outpace height. That is, $\mathbf{F}$ makes it a condition on sethood that the images $y$ of any mapping $\psi(x, y)$ on a genuine set $A$ must be bounded in rank and hence, eventually, must all be available to be collected into a set at some stage, as indicated in Figure 3. F, that

of atoms without attempting to accommodate wide sets. This is the picture described by Friedman (2004), who argues that there are strong reasons for including $\mathbf{A}_{\infty}$ as an axiom of ZFCU.

${ }^{17}$ The stage theories of Boolos (1971), Scott (1974), Van Aken (1986), Uzquiano (2002), and Potter $(2004,225 \mathrm{ff}, 296 \mathrm{ff})$ that are meant to formalize the iterative conception proper all fail to entail $\mathbf{F}$ without the addition of formal principles that are similar in spirit to, and at least as strong as, F. See Hallett 1984, 219-223 for additional commentary on the stage theories of Boolos and Scott. Shoenfield (1967, 238ff) develops an informal account of the iterative conception that includes a very strong "confinality" principle that appears to entail F. Hallett $(1984,217-218)$ argues convincingly that this principle is itself in need of at least as much justification as F, a claim well-confirmed by Reinhardt's (1974) reconstruction of Shoenfield's account, where his representation of the cofinality principle yields not only $\mathbf{F}$ but the existence of a measurable cardinal. 


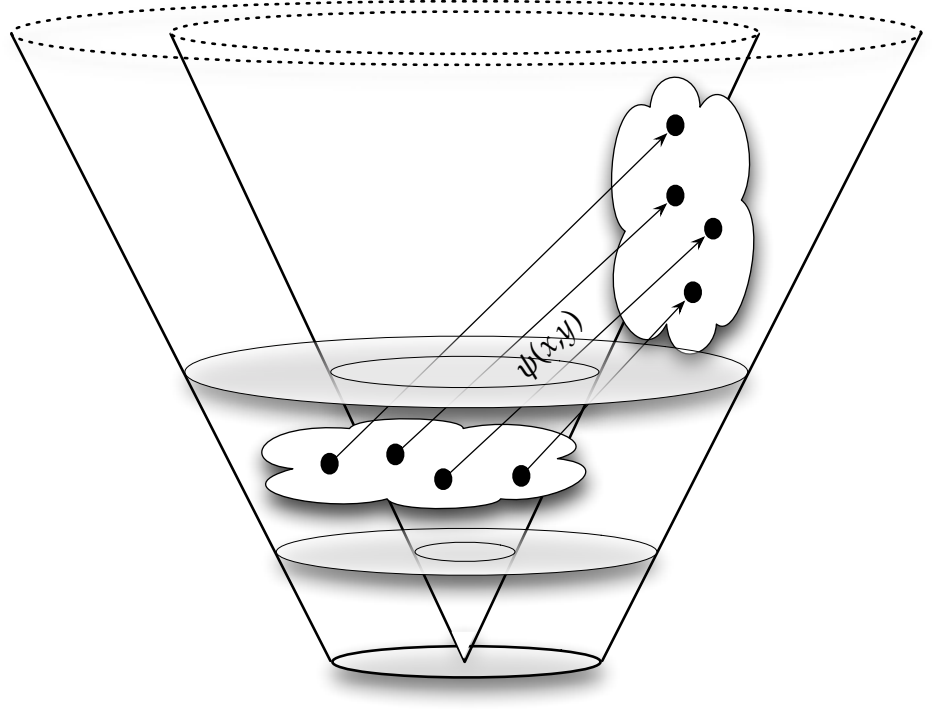

Figure 3: Replacement in ZFCU

is to say, forces narrowness into the concept of set contrary to the iterative conception.

And thus the disconnect: If in fact the set theoretic universe has the structure of Figure 2 rather than Figure 1, an application of $\mathbf{F}$ to sets that are at least as wide as the universe is high could cause us to "replace" our way out of the universe; it could enable us to prove the existence of sets that are, in fact, "too high" to be formed at some stage of the cumulative hierarchy, as depicted in Figure 4. This would of course lead quickly to contradiction.

\subsection{First Cut at a Solution}

A hasty solution is just to abandon Replacement altogether. That would obviously prevent the scenario of Figure 4, but at high cost. True enough, Replacement is arguably stronger than is warranted directly by the iterative conception. But the central purpose of set theory is not to axiomatize the iterative conception but to explore the universe of sets, and that universe is greatly underdetermined by the iterative conception. A fruitful exploration must therefore incorporate axioms that take us beyond the basic principles that the iterative conception directly warrants, axioms that 


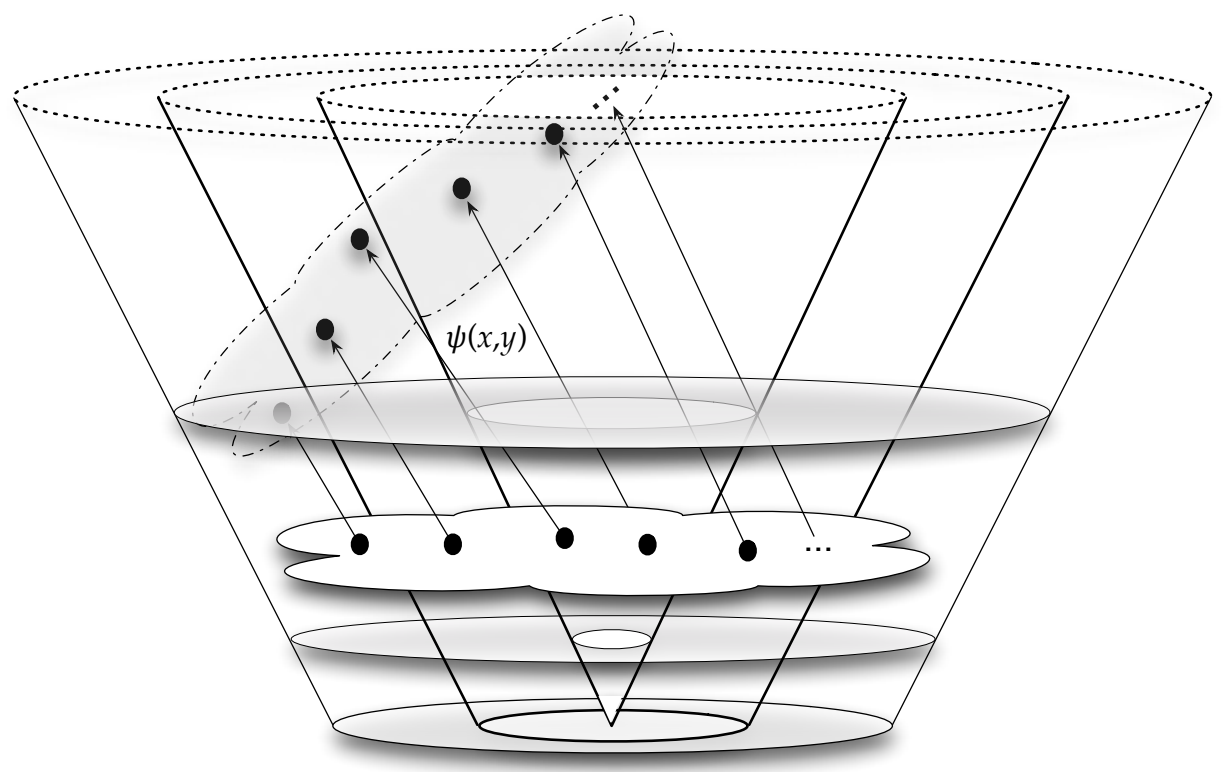

Figure 4: $\mathrm{F}$ in the context of wide sets

derive their justification from some combination of intuitive "naturalness" (relative to the iterative conception) and theoretical utility - which, in the case of Replacement, is substantial. Throwing Replacement out simply to accommodate wide sets would therefore be unnecessarily Draconian.

A gentler and more fruitful solution is simply to restrict Replacement to the determinable sets. Determinability is naturally defined as the property $\exists \kappa x \approx \kappa$ of being as large as some cardinal. However, following Menzel 1986, we will opt instead for an equivalent definition (in ZFCU) that both relies on a bit less machinery (notably, it does not presuppose AC) and draws on somewhat more general intuitions about sets and size rather than number per se. Specifically, we will look instead to the "inner core" of pure sets within the cumulative hierarchy. Within that core, all sets are "narrow", as we begin only with the empty set — as narrow a set as there could be - and build sets by means of operations (including Replacement) that are assumed only to yield narrow sets from narrow sets. In the presence of wide sets, then, Replacement cannot get us into trouble so long as it only applies to sets that are no larger than any pure set.

Accordingly, define a set to be narrow, or mathematically determinable, if it is as large as some pure set: 


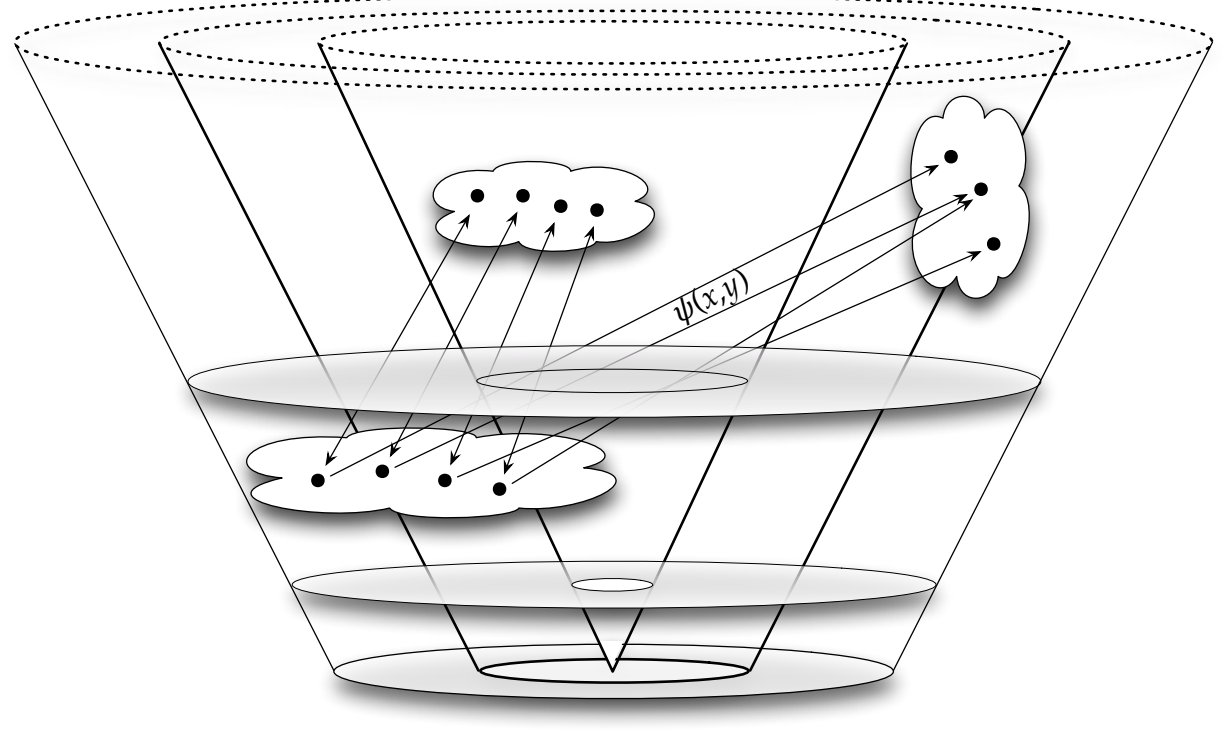

Figure 5: Replacement restricted to determinable sets

$$
\operatorname{Det}(x) \equiv_{d f} \operatorname{Pure}(x) \vee \exists y(\operatorname{Pure}(y) \wedge x \approx y) \cdot{ }^{18}
$$

We then restrict Replacement accordingly:

$$
\mathbf{F}^{\prime} \operatorname{Det}(A) \rightarrow(\forall x \in A \exists ! y \psi \rightarrow \exists B \forall y(y \in B \leftrightarrow \exists x \in A \psi)){ }^{19}
$$

The condition that a given set must be measured against the pure sets before $\mathbf{F}^{\prime}$ can be applied, as depicted in Figure 5, thus banishes the specter of replacing our way out of the universe.

\footnotetext{
${ }^{18}$ The left disjunct here might seem redundant, as every set $A$ is obviously as large as itself - notably, for every set $A$ there is the identity bijection $f: A \longrightarrow A$ where $f=\{\langle a, a\rangle: a \in A\}$. Hence, every pure set is determinable in virtue of the right disjunct alone. However, proving the existence of the identity function on a set $A$ generally - a subset of $A \times A-$ requires either Powerset or Replacement, and we will be modifying those axioms in ways that require that at least some pure sets - notably, the ordinals be provably determinable independently of them; see the following footnote.

${ }^{19}$ The Pure predicate was not defined in terms of a transitive closure function in $\S 1.2$ in anticipation of $\mathbf{F}^{\prime}$, as that function is (typically) defined by recursion on $\omega$, and the proof that such definitions are legitimate in general requires Replacement; since $\mathbf{F}^{\prime}$ presupposes that Pure is well-defined, we would thus have been in danger of a vicious circle. Defining Pure in terms of the relational notion TC, however, enables us to prove that the ordinals are pure, hence determinable, without Replacement. This, in turn, enables us to use $\mathbf{F}^{\prime}$ to prove that definitions by recursion on $\omega$, and indeed any ordinal, are legitimate without any risk of circularity.
} 
$\mathbf{F}^{\prime}$ obviously generalizes $\mathbf{F}$. However, the proof of $\sim \mathbf{S o A}_{\infty}$ in $\S 1.3$ breaks down if we replace the latter with the former. ${ }^{20}$ Like the iterative conception itself, then, the resulting theory is silent on the matter of how many urelements there are. ${ }^{21}$ We thereby break the dubious connection between sethood and size that is forced upon us by the standard Replacement schema $\mathbf{F}$.

This revision does bring one complication with it. Fraenkel's Replacement schema $\mathbf{F}$ is critical to establishing the legitimacy of definitions by recursion on $\in$ and thereby to the theorem that every set has a rank, that every set is formed at some ordinal level in the iterative process. However, the use of Replacement in this theorem depends critically upon the limitation of size assumption that is built into $\mathbf{F}$. That assumption has been removed in $\mathbf{F}^{\prime}$, so we cannot hope to use it to demonstrate the legitimacy of the usual definition of a general rank function that can apply to both narrow and wide sets alike. ${ }^{22}$

Accordingly - and, arguably, fittingly, given the fundamental conceptual role that the idea of a well-ordered hierarchy of "levels" plays in the intuitive underpinnings of the iterative conception - we simply introduce the ' $r n k$ ' function symbol as a primitive of our language and take its usual definition Rnk above to be an independent axiom of the system. With this axiom, then, along with the failure of (1), we ensure that rank, rather than possession of a definite cardinality, is the definitive characteristic of iterative sethood.

Let ZFCU' be the result of replacing $\mathbf{F}$ in ZFCU with $\mathbf{F}^{\prime}$ and adding $\mathbf{R n k}$ as an axiom.

\footnotetext{
${ }^{20}$ Specifically, the proof breaks down because it relies on (1), whose proof requires that $\mathbf{F}$ be applicable to all sets. The proof in $\mathrm{fn} 12$ of $\sim \mathbf{S o A}_{\infty}$ in ZFU also breaks down if $\mathbf{F}^{\prime}$ replaces $\mathbf{F}$ because, in the proof of Hartogs' theorem, there is nothing to guarantee that the set $C$ of well-orderings of subsets of the initial set $A$ is determinable if $A$ itself isn't.

${ }^{21}$ Zermelo (1930) himself built the essential "narrowness" of sets into his formal development of the cumulative hierarchy. McGee $(1997,51)$ follows Zermelo on this point.

${ }^{22}$ Indeed, it is fairly easy to see how the definition might break. Say that a set $A$ is hereditarily impure if $\varnothing$ is not in its transitive closure, i.e., equivalently, if every set in its transitive closure is impure. If we weaken $\mathbf{F}$ to $\mathbf{F}^{\prime}$, our axioms would not rule out a universe containing hereditarily impure sets of arbitrarily high rank and, hence, sets which themselves lack a rank. In this model, that is, the universe continues to grow "beyond" the ordinals and no sets that are part of this new growth, therefore, have a rank.
} 


\subsection{Number, Powerset, and the Absolutely Infinite}

The consistency of ZFCU' with SoA $\mathbf{A}_{\infty}$ notwithstanding, modifying only the Replacement schema and leaving ZFCU in other respects untouched leads to awkward consequences for the notion of number. This is most obviously the case with the Powerset axiom PS. For if we postulate a wide set $A^{*}$ of atoms, then, by Cantor's Theorem, its powerset $\wp\left(A^{*}\right)$ will be strictly larger than $A^{*}, A^{*}<\wp\left(A^{*}\right)$; likewise $\wp\left(A^{*}\right)<\wp\left(\wp\left(A^{*}\right)\right)$, and so on. But since (1) fails in ZFCU', so too does the definition of the cardinality operator; in particular, $A^{*}, \wp\left(A^{*}\right), \wp\left(\wp\left(A^{*}\right)\right), \ldots$ in ZFCU' all lack a definite cardinality. But what else does a progression of propositions of the form $A<B, B<C$, ... indicate than a progression of increasing sizes? And what else can such increases in size be but increases in cardinality?

There is a natural response to this situation with a strong historical precedent. It is widely acknowledged now that, even in the earliest developments of his theory of transfinite numbers, Cantor recognized that some collections - notably, the collection of all ordinal numbers - are themselves "too big" to be assigned a definite size, a definite cardinal number. This idea led to the development of the limitation of size approach to the set theoretic paradoxes that focuses on over-largeness per se as their source. I am of course strongly challenging that view - it is only when a collection is over-large due to unboundedness that paradox can arise. However, it seems to me it is quite intuitive to say that the "size" of a set that cannot be measured by the cardinals that emerge in the course of the set formation process represents an unsurpassable - albeit mathematically indeterminable - limit, what Cantor called the "absolutely infinite": an "absolute quantitative maximum" that is larger than any set with a definite cardinality and is incapable of either determinable measure or any definite form of increase; no two absolutely infinite sets can differ in size in any mathematically definite sense. ${ }^{23}$

In $\mathrm{ZFCU}$, then, the absolutely infinite isn't really absolute in the Can-

\footnotetext{
${ }^{23}$ See Cantor 1967, 116 Cantor 1932, esp. 375, 404-405, Menzel 1984, Hallett 1984, $12 \mathrm{ff}$ and Jané 1995. It should be noted that, although Cantor often talks as though absolutely infinite collections are sets (and indeed occasionally refers to them as Mengen), in his later thought he held (e.g., 1967, 114) that an absolutely infinite collection can't be thought of as "one finished thing", i.e., it would seem, as a set in the fullest sense of the term. I would therefore not want to suggest that I am spelling out a conception of set that Cantor himself clearly had in mind.
} 
torian sense: because Cantor's theorem still holds, every absolutely infinite set is smaller - in a completely rigorous mathematical sense - than some other absolutely infinite set. Moreover, the existence of such determinate distinctions in size seems to suggest that one has simply not allowed the set formation process to continue far enough to generate the cardinal numbers necessary for representing these distinctions. Of course, any attempt to extend the hierarchy to generate larger cardinals will encounter the same difficulty anew on the assumption of an indeterminable set of atoms. Hence, ZFCU' appears to reflect a structurally untenable picture of the set theoretic universe.

To correct this defect, then, the universe must be so represented by our axioms that they do not subject the absolutely infinite to any sort mathematically definite increase through Cantor's Theorem. One way to accomplish this, of course, is to follow $\mathbf{F}^{\prime}$ in restricting the Powerset axiom to narrow sets. But that is a strong restriction; for example, if $A$ is wide, it blocks the usual proof that $A$ has a Cartesian product $A \times A$. But the restriction is in fact needlessly strong; it is enough to place the determinability restriction, not on the applicability of the axiom, but on its output: given a set $A$, all the determinable subsets of $A$ constitute a further set:

\section{PS $^{*} \exists B \forall x(x \in B \leftrightarrow \operatorname{Det}(x) \wedge x \subseteq A)$.}

In particular, where $\wp^{*}$ is the "determinable" powerset operation given by PS $^{*}$, we are still able to derive $A \times A$ as a subset of $\wp^{*}\left(\wp^{*}(A)\right)$, for any set $A$.

PS $^{*}$ is obviously equivalent to PS under the assumption that all sets are determinable; but without that assumption Cantor's Theorem cannot be proved $^{24}$ and, hence, the revised axiom does not allow us to demonstrate any definite distinctions in size between absolutely infinite sets. Furthermore, it cannot be shown any longer that the stages themselves constitute sets at all. For while all possible sets of atoms are formed at the very first stage, PS $^{*}$ only guarantees that determinable collections of them are themselves sets.

With this modification, then, there is no provable "expansion" of the cumulative hierarchy from stage to stage. But shouldn't we say more? PS*

\footnotetext{
${ }^{24}$ Specifically, where $g$ is a function from $A$ to $\wp^{*}(A)$, if $A$ is not determinable, the diagonal set $\{x \in A: x \notin g(x)\}$ for $g$ cannot be shown to be determinable and, hence, may not be in $\wp^{*}(A)$. This leaves open the possibility that $g$ is onto.
} 
is only compatible with the idea of a maximal absolutely infinite; it does not express it. Since it is only a weakening of PS, for all the axiom tells us, there could still be definite size differences within the absolutely infinite. To rule this out, an additional axiom is needed. One possibility is to assert explicitly that all indeterminable sets are the same size:

$\operatorname{Max}(\neg \operatorname{Det}(A) \wedge \neg \operatorname{Det}(B)) \rightarrow A \approx B$

However, by my lights, this axiom itself constitutes an unjustifiably definite fact about the absolutely infinite - from the fact that there is no mathematically definite increase within the absolutely infinite, it does not obviously follow that distinct absolutely infinite sets should be explicitly comparable, i.e., that there should exist a bijection between any two of them. I therefore suggest the more modest axiom that a definite distinction in size between sets can exist only when the smaller of the two is determinable:

Det $A<B \rightarrow \operatorname{Det}(A)$.

Although weaker than Max, Det nonetheless prevents the sort of definite size differences between absolutely infinite sets that are provable in ZFCU'. On the assumption of a wide set of atoms, then, we are led to a rather different, "cylindrical" picture of the universe, as depicted in Figure $6 .{ }^{25}$ For, as the universe is absolutely infinite from the start on that assumption, under PS $^{*}$, although infinitely many new sets appear at each stage of the cumulative hierarchy, there is no mathematically definite increase in its "girth".

\subsection{Likewise Choice?}

Considerations similar to those in the preceding section might suggest that a corresponding modification of $\mathrm{AC}$ is warranted. For suppose the set $A^{*}$ of atoms is indeterminable and, hence, absolutely infinite. Then if we place no restrictions on Choice, there is a well-ordering $R$ of $A^{*}$. Reasoning analogously to the above, since $A^{*}$ is absolutely infinite, the "order type" of $R$

\footnotetext{
${ }^{25}$ Figure 6 is a bit misleading insofar as it suggests that the growth of the hereditarily determinable sets will eventually catch up to and, indeed, outpace the hereditarily indeterminable sets, but this is of course only a side-effect of the author's limited skills as a graphic artist.
} 


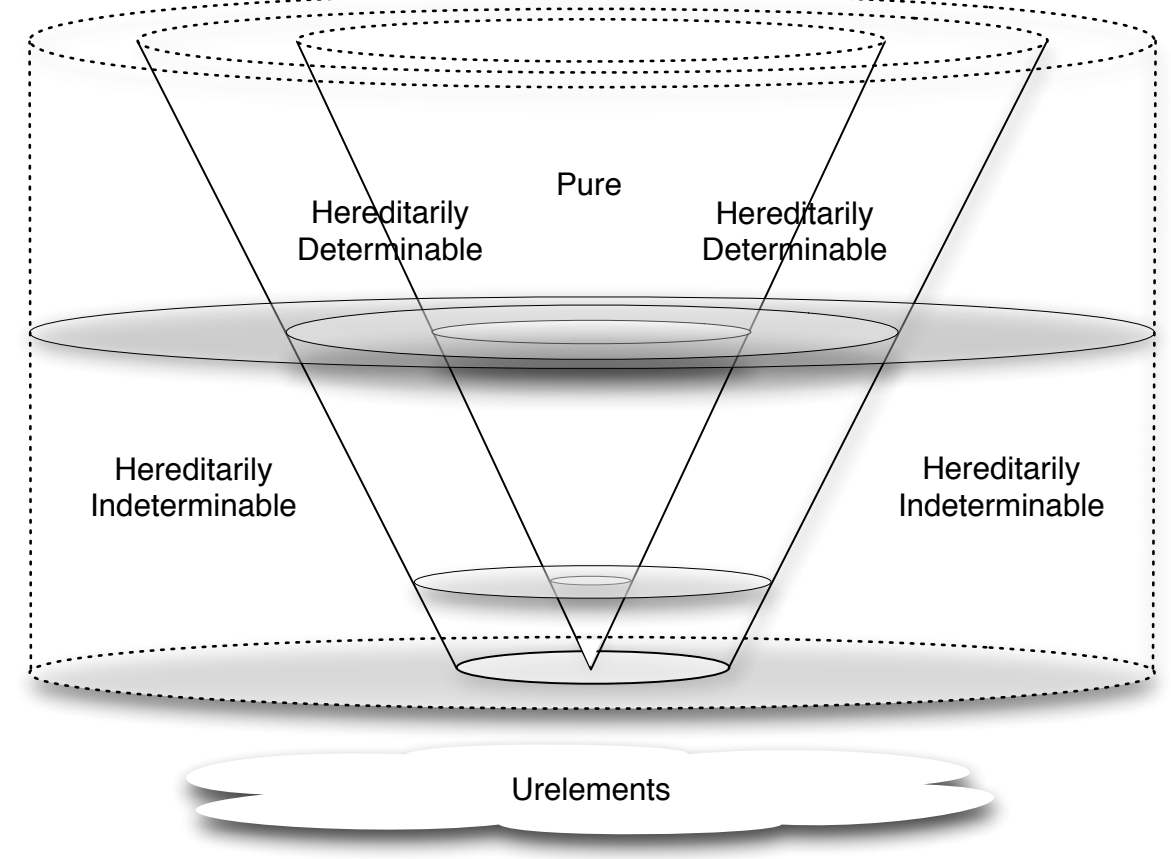

Figure 6: The cumulative hierarchy with absolutely infinite sets

should represent an immeasurable ordinal maximum. But let $a_{0}$ be the $R$ least element of $A^{*}$ and define $R^{+}$such that, for $x, y \in A^{*}, R^{+}(x, y)$ iff $x \neq a_{0}$ and either $R(x, y)$ or $y=a_{0}$. ( $R^{+}$, that is, is just like $R$ except that $a_{0}$ is now " $R^{+}$-greater than" all other atoms.) Since we can map $\left\langle A^{*}, R\right\rangle$ isomorphically into $\left\langle A^{*}, R^{+}\right\rangle$but not vice versa, $\left\langle A^{*}, R\right\rangle$ is intuitively "shorter" than $\left\langle A^{*}, R^{+}\right\rangle$; indeed, intuitively, the "order type" of the latter is the "successor" of the "order type" of the former. But, analogous to the situation with Powerset, there are no corresponding ordinals to represent this fact and this, in turn, warrants restricting $\mathrm{AC}$ to determinable sets.

There is a flaw in this reasoning, however. For the general proof that, given AC, an arbitrary set $A$ is well-orderable constructs a well-ordering by means of a choice function on $\wp(A) \backslash\{\varnothing\}$. But PS* yields only the set $\wp^{*}(A)$ of determinable subsets of $A$ and, hence, in concert with AC, guarantees only that determinable sets are well-orderable. So the inference from AC to the well-orderability of $A^{*}$ in the argument above fails.

I believe this comports well with our reasoning thus far. Intuitively, the powerset operation "generates" new levels of the iterative hierarchy and, 
hence (as we've seen and as we will argue further shortly), the extent of its ability to do so can rightly be questioned when it leads to conflict with other intuitions. By contrast, AC does not involve any upward growth of the hierarchy; given a choice-friendly set $A, \mathbf{A C}$ only postulates the existence of a set $C_{A}$ of members of members of $A$ and thus a set whose members all already exist "prior" to $A$ in the hierarchy. Moreover, $C_{A}$ is no larger than $A$. Hence, on iterative grounds $C_{A}$ "ought" to exist and there are no overriding considerations of size that emerge from the assumption that it does. Thus, there seem to be no principled objections to AC in the context of wide sets beyond standard qualms about its nonconstructive character and its allegedly unintuitive consequences. ${ }^{26}$

\subsection{Replacement Redux}

If $A$ is an indeterminable set, our modified axioms $\mathbf{P S} \mathbf{S}^{*}$ and $\mathbf{F}^{\prime}$ leave us without the ability to prove the existence of certain intuitively unproblematic sets. For example, for atoms $a$, let $A_{a}^{*}$ be $A^{*} \backslash\{a\}$; then, as things stand, we are unable to prove that there is a set $\left\{A_{a}^{*}: a \in A^{*}\right\}$ comprising the values of the mapping $a \mapsto A_{a}^{*}$ on $A^{*}$. But these values are all "constructed" along with $A^{*}$ at the very first stage and, hence, are "available" to form a set at the next stage; moreover, there are no more of them than there are atoms to conflict with our conception of the absolutely infinite. So, as with choice sets, there seems no principled reason to deny that the $A_{a}^{*}$ constitute a set.

Fortunately, a bit of reflection reveals that the modification of Replacement that gave us $\mathbf{F}^{\prime}$ - restricting Replacement's applicability to determinable sets - is in fact unnecessarily strong. The purpose of the restriction was to satisfy the central structural constraint on set formation, namely, boundedness in rank. Since $\mathbf{F}^{\prime}$ is precisely what guarantees that the range

\footnotetext{
${ }^{26}$ See, e.g., Fraenkel et al. 1973, 80-86. It is chiefly for these reasons that I have opted for $\mathrm{AC}$ rather than its ZF-equivalent that every set of nonempty sets has a choice function:

ACF $\forall x \in A \exists y y \in x \rightarrow \exists f \forall x \in A f(x) \in x$.

For while a choice function for a set $A$ is of no greater cardinality than $A$, it is of higher rank than $A$. Hence, it involves us in questions concerning the nature of the upward growth of the hierarchy beyond $A$ which, as we've argued, requires careful consideration when $A$ is indeterminable. The existence of choice functions thus seems neither as immediate nor as intuitively unproblematic as the existence of choice sets. (That said, ACF is in fact provable in our final theory $\mathrm{ZFCU}{ }^{*}$.)
} 
of any mapping on a determinable set is bounded in rank, the restriction in question is obviously sufficient for satisfying this structural constraint. But it isn't necessary. For suppose we don't know if a given set $A$ is determinable, or we even know flat out that it is not, but that we can also show independently that the range of a given (functional) mapping $\psi$ on $A$ is bounded in rank; then the structural constraint is again satisfied. And because the functionality of the mapping guarantees the range is no larger than $A$, there is no reason to deny that the range of the mapping constitutes a set.

More precisely, then, say that $\psi$ (in which, typically, ' $x$ ' and ' $y$ ' occur free) is bounded above on a set $A, B A(\psi, A)$, just in case such a bound exists:

$$
B A(\psi, A)=d_{f} \exists \alpha \forall x \in A(\psi \rightarrow r n k(y)<\alpha) .{ }^{27}
$$

And we now say that the range of a mapping $\psi$ on a set $A$ constitutes a set so long as $A$ is either determinable or $\psi$ is bounded above on $A$ :

$$
\mathbf{F}^{*}(\operatorname{Det}(A) \vee B A(\psi, A)) \rightarrow(\forall x \in A \exists ! y \psi \rightarrow \exists B \forall y(y \in B \leftrightarrow \exists x \in A \psi)) .
$$

So revised, our schema now applies to determinable and indeterminable sets alike but enforces, not a limitation of size, but a more general limitation of height on the collections that can count as sets, as desired. ${ }^{28}$ We depict the effect of $\mathbf{F}^{*}$ in our "cylindrical" universe of sets in Figure 7.

To illustrate the use the axiom with the example above: By the rank axiom Rnk, for atoms $a, \operatorname{rnk}(a)=0$, so $\operatorname{rnk}\left(A_{a}^{*}\right)=1$. Hence, the formula ' $y=$ $A_{x}^{*}$ is bounded above on $A^{*}$ by 2 so, by $\mathbf{F}^{*}$, the set $\left\{y: \exists x \in A^{*} y=A^{*} \backslash\{x\}\right\}=$ $\left\{A_{a}^{*}: a \in A^{*}\right\}$ exists. $^{29}$

\footnotetext{
${ }^{27}$ Note we cannot replace the determinability condition of $\mathbf{F}^{\prime}$ with this condition. For we need to be able to prove all of the usual ordinals of ZFC exist independently of the boundedness condition to have them available for applying the condition. See also fns 19.

${ }^{28}$ van Aken $(1986,1003)$ comes very close to expressing the idea underlying $\mathbf{F}^{*}$ : "For a set to exist, it suffices that the set either is small enough or has elements of bounded rank." However, this idea (which is formalized in van Aken's schema RC of rank comprehension (ibid., 995)) is stronger than $\mathbf{F}^{*}$, since as it guarantees that any collection of things of bounded rank is a set, whereas $\mathbf{F}^{*}$ in effect requires a demonstration that, for any bounded collection of things, there are no more of those things than there are members of some (possibly wide) set. As a consequence, stages in the iterative hierarchy themselves turn out to be sets in van Aken's theory and so (given Choice) determinability and boundedness in rank turn out to be coextensive. So, in the end, despite the appearance of a distinction between determinability and boundedness in rank in van Aken's quote above, the distinction collapses in his theory.

${ }^{29}$ Spelling out ' $y=A_{x}^{*}$ ' a bit more correctly: ' $\exists A(\forall z(z \in A \leftrightarrow \sim \operatorname{Set}(z)) \wedge y=A \backslash\{x\})$.
} 


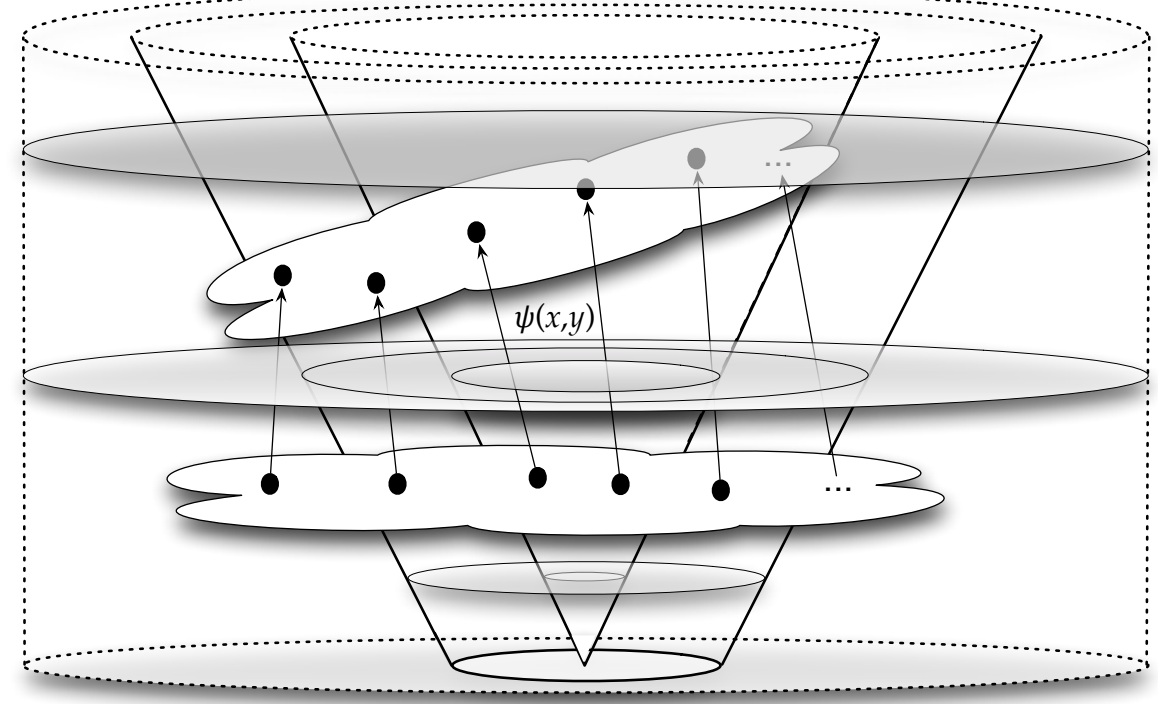

Figure 7: $\mathbf{F}^{*}$ in the cumulative hierarchy

Let $Z$ FCU ${ }^{*}$ be the result of replacing $\mathbf{F}$ with $\mathbf{F}^{*}$ and $\mathbf{P S}$ with $\mathbf{P S}{ }^{*}$ in $\mathrm{ZFCU}$ and adding Rnk and Det as axioms. ZFCU* is obviously consistent if ZFCU is, since $Z F C U^{*}+\forall A \operatorname{Det}(A)$ just collapses into ZFCU (plus appropriate definitions). However, unlike ZFCU, ZFCU* is consistent with SoA $_{\infty}$ (relative to an extension of $Z F C-a$ proof is sketched in the Appendix). And, unlike ZFCU', ZFCU* avoids the disconnect between relative size and the possession of a definite cardinality.

\subsection{Are We Any Better Off?}

Perhaps the strongest objection to ZFCU* is this: ZFCU*, in particular PS*, reintroduces the same sort of tensions with the iterative conception that motivated our project in the first place. For suppose $\operatorname{SoA}_{\infty}$, i.e., that, at the very first stage of set formation, we find a wide set $A^{*}$ of atoms. Intuitively, at that same stage, every other set of atoms is also formed. Hence, at that stage all of $A^{*}$ 's subsets are all there to be collected, just as all the atoms are prior to the first stage. Hence, the set of all those subsets should be formed at the very next stage. Thus, one might argue, there is no genuine 
philosophical win here; the same tension with the iterative conception that arises for ZFCU vis-á-vis a set of all atoms arises straightaway "one level up" for ZFCU* vis-á-vis a set of all sets of atoms.

First, as noted briefly in the following section, from a more pragmatic point of view, allowing a set of atoms has its advantages for philosophical applications. That aside, it is still far from clear that the two situations are on a par. The iterative conception tells us that, at a given stage, all the sets exist that can be formed from the objects in preceding stages - call this the iterative intuition. The full power set axiom PS is but one possible formal expression of this intuition and not obviously the most fitting. For, in fact, while our grasp of the axiom is strong and intuitive for finite sets, it is tenuous with regard to infinite sets. Notably, by a well known result of Easton (1970), beyond Cantor's Theorem, we have no clear grasp of the effects of the full powerset operation on infinite sets; in particular, there is basically no upper bound on how large the power set $\wp(\mathbb{N})$ of the set $\mathbb{N}$ of natural numbers might be. Indeed, so little is known of the behavior of full powerset that Hallett $(1984,208)$ is driven to conclude that the operation "is just a mystery".

Powerset is thus both extremely powerful and extremely mysterious, and its mysteries suggest the need for some measure of theoretical justification for determining the extent to which its unrestricted applicability is the proper expression of the iterative intuition. Its applicability to finite sets is obviously warranted, as full powerset just follows from logic. Beyond this, the existence of our two most fundamental number systems, $\mathbb{N}$ and $\mathbb{R}$, and the striking relation between them - that $\mathbb{R} \approx \wp(\mathbb{N})-$ provides compelling warrant for the applicability of full powerset to countable sets. Higher analysis in turn warrants its applicability to sets the size of the continuum. More generally, and more liberally, there seems to be no compelling reason to restrict its applicability to sets already generated from unrestricted powerset as we make our way up the ordinal backbone of the pure cumulative hierarchy $V$ and, therefore, to sets the same size as any of those sets. We seem, therefore, to have a consistent general justification for the applicability of Powerset to determinable sets. Finally, if somewhat more tentatively, as it leads to no conflicts with our notions of size and number, we can generalize one last step to the formation of the determinable subsets of any given set into a further set - i.e., to PS*. 
It is the examples of $\mathbb{N}$ and $\mathbb{R}$, then, and the relation between them that gets the justification of $\mathbf{P S}^{*}$ off the ground. However, we have no analogous examples of a powerset-based relation between indeterminable sets; indeed, we have found strong arguments for the idea that progressions of increasing size should also reflect relations between cardinals and, hence, that there should be no definite differences of size among indeterminable sets.

Given these reflections, then, as I see it, the burden for justification lies not on our "restricted" Powerset axiom PS*, for which we can develop a comprehensive justification with regard to determinable and indeterminable sets alike based both upon the iterative intuition and actual structures out of mathematical practice, but rather on its unrestricted counterpart PS, for which we cannot find a parallel justification. PS*, rather than full PS, appears on reflection to be the more warranted expression of the iterative intuition.

\section{Summary and Final Reflection}

The proposition SoA $\mathbf{A}_{\infty}$ that there is a wide set of atoms - a set of bounded rank but indeterminable size - is intuitively consistent with the iterative conception of set but formally inconsistent with ZFCU, a disconnect traced to the Replacement schema $\mathbf{F}$. A simple modification $\mathbf{F}^{\prime}$ of $\mathbf{F}$ led us to a theory $\mathrm{ZFCU}^{\prime}$ that is consistent with $\mathbf{S o A}_{\infty}$ and, hence, arguably more faithful to the iterative conception than ZFCU. However, ZFCU' introduces an uncomfortable new disconnect between the notions of relative size and number: as full Powerset PS is an axiom of ZFCU', there are still definite gradations of size among wide sets, their lack of definite cardinalities notwithstanding. Cantor's intuition that the absolutely infinite should not be capable of determinate increase motivated a restriction on Powerset that only yields a set comprising the "narrow" subsets of a given set. Combined with a strengthening $\mathbf{F}^{*}$ of $\mathbf{F}^{\prime}$, we arrived at a theory $\mathbf{Z F C U ^ { * }}$ that is consistent with the existence of wide sets but which seems to preserve Cantor's intuition. Finally, we argued that the restrictions on full powerset in $\mathbf{Z F C U}^{*}$ can be reconciled with the central intuition underlying the iterative conception of set.

Our work here was prompted initially by an observation concerning Lewis's modal realism. So it would be appropriate to note very briefly the 
major implications of our results for Lewis's metaphysics. Because Lewis needs arbitrary sets of atoms in his theory to serve as properties and propositions, and arbitrary sets of pairs of atoms to serve as relations (notably, counterpart relations), the argument from the principle of Recombination $\mathbf{R}$ to $\mathbf{A}_{\infty}$ led him to reject $\mathbf{R} \cdot{ }^{30}$ As that argument depends on SoA $\mathbf{A}_{\infty}$ 's inconsistency with ZFCU, the argument fails if ZFCU is replaced by either of the theories developed here; in both, one can quantify freely over properties, propositions, and relations. The set $\wp^{*}\left(A^{*}\right)$ of all properties in our philosophically preferred theory ZFCU* is somewhat thinner than the full powerset; the possible limitations of this for the modal realist is left as a matter for further investigation. However, as $\mathbf{P} \mathbf{S}^{*}$ and $\mathbf{F}^{*}$ together permit the construction of infinitely many complex sets of arbitrarily high rank over $A^{*}$, wide and narrow alike, it is conjectured that $\mathrm{ZFCU}{ }^{*}$ provides the modal realist with all the set theory needed for philosophical applications of possible worlds. “"

\section{Appendix: Relative Consistency Proofs}

Let $\mathrm{ZFC}^{+}$be $\mathrm{ZFC}+$ "There is an inaccessible cardinal". We show that both ZFCU' and $\mathrm{ZFCU}^{*}$ are consistent with the existence of a wide set of atoms relative to $\mathrm{ZFC}^{+}$. Let $\kappa$ be the least inaccessible and let $A^{*}=\{\langle\kappa, \alpha\rangle: \alpha<\kappa\}$. $A^{*}$ will serve as the set of atoms for our models.

A Model of ZFCU' + SoA $_{\infty} \cdot$ For $\alpha<\kappa$ and limit ordinals $\lambda \leq \kappa$, let

$$
\begin{aligned}
U_{0} & =A^{*} \\
U_{\alpha+1} & =U_{\alpha} \cup \wp\left(U_{\alpha}\right) \\
U_{\lambda} & =\bigcup_{\alpha<\lambda} U_{\alpha}
\end{aligned}
$$

Let $U=U_{\kappa}$. To facilitate matters, let $\rho_{U}: U \longrightarrow \kappa$ be the obvious "rank" function for $U: \rho_{U}(x)=\sup ^{+}\left\{\rho_{U}(y): y \in U \wedge y \in x\right\}$. ${ }^{32}$ Now, as definitions can be understood as axioms that conservatively extend a given theory, it is

\footnotetext{
${ }^{30}$ See 1986, 103-104 and Forrest and Armstrong 1984.

${ }^{31}$ Lewis himself explored an alternative approach to set theory in his 1991.

${ }^{32}$ Note, importantly, since $\langle\kappa, \alpha\rangle=\{\{\kappa\},\{\kappa, \alpha\}\}$, neither $\{\kappa\} \in U$ nor $\{\kappa, \alpha\} \in U$ so we have $\rho_{U}(\langle\kappa, \alpha\rangle)=0$ for the "atoms" $\langle\kappa, \alpha\rangle$ of our model.
} 
easiest to consider the theory that results from adding TC, Pure, Det, and rnk to the language of ZFCU' and extending ZFCU' with the corresponding definitions above. (We can thus suppose that the antecedent 'Det $(A)^{\prime}$ of $\mathbf{F}^{\prime}$ is initially spelled out in the primitive terminology of ZFCU'.) Accordingly, first, we define a "transitive closure" function on $U$ in terms of an auxiliary function $g: U \times \omega \longrightarrow U:^{33}$

$$
\begin{aligned}
g(x, 0) & =x \\
g(x, n+1) & =\bigcup\left(g(x, n) \backslash A^{*}\right) \cup g(x, n)
\end{aligned}
$$

Thus, given $g(x, n), g(x, n+1)$ holds on to everything in $g(x, n)$ and adds to it the union of all the "sets" in $g(x, n)$. (The "atoms" of our model have to be excluded from the application of the union operator, of course, because they are in fact themselves sets of the form $\{\{\kappa\},\{\kappa, \alpha\}\}$.) Given $g$, we define our transitive closure function as the closure of $g:{ }^{34}$

$$
t c_{U}(x)=\bigcup_{n<\omega} g(x, n)
$$

Now define the interpretation $\mathcal{M}=\langle U, I\rangle$ of the (extended) language of ZFCU' such that:

- $I\left({ }^{\prime} \epsilon^{\prime}\right)=\{\langle a, b\rangle \in U \times U: a \in b\}$

- $I\left({ }^{\prime}\right.$ Set $\left.^{\prime}\right)=U \backslash A^{*}$

- $I\left({ }^{\prime} T C^{\prime}\right)=\left\{\langle a, b\rangle: a \in U \backslash A^{*} \wedge b=t c_{U}(a)\right\}$

- $I\left({ }^{\prime}\right.$ Pure' $)=\left\{A \in U: \forall a \in t c_{U}(A) a \notin A^{*}\right\}$

- $I\left({ }^{\prime}{ }^{2 e t}\right)=\{A \in U:|A|<\kappa\}$

- $I\left({ }^{\prime} r n k '\right)=\rho_{U}$.

It is obvious that Rnk is true in $\mathcal{M}$ and, as the set $A^{*}$ of "atoms" is an indeterminable set in $\mathcal{M}$, it is easy to see that $\mathbf{A}_{\infty}$ (as spelled out formally in

\footnotetext{
${ }^{33}$ The idea behind the inductive clause in the definition here is that $\bigcup\left(g(x, n) \backslash A^{*}\right)$ prevents "urelements" $\left\langle\kappa^{*}, \alpha\right\rangle$ from being "decomposed" by the union operator; and the purpose of $\left(g(x, n) \cap A^{*}\right)$ is to enable $g$, at each level $g(x, n+1)$, to carry along the "urelements" that were revealed in the preceding level.

${ }^{34}$ Because sets are well-founded eventually for some $n, g(x, m)=\varnothing$ for all $m>n$.
} 
$\S 1.3)$ and SoA are true in $\mathcal{M}$ as well. ${ }^{35}$ Moreover, since $\mathcal{\kappa}$ is a limit ordinal $>\omega$, it is straightforward to verify that all of the axioms of $\mathrm{ZU}+\mathbf{A C}$ are also true in $\mathcal{M}$.

The only less than obvious case is the Replacement schema $\mathbf{F}^{\prime}$. We first note the following easily verified

Fact: For $x \in U$, if $\rho_{U}(x)<\alpha$, then $x \in U_{\alpha}$.

So suppose $A \in U \backslash A^{*}$ and that $A$ is "determinable" in $\mathcal{M}$, i.e., that $|A|<\kappa$, and that $\psi$ is a formula that, relative to some variable assignment $s$, satisfies the antecedent of the consequent of $\mathbf{F}^{\prime}$. That is, where $\psi_{s}^{\mathcal{M}}=\{\langle a, b\rangle$ : $\left.\mathcal{M}, s_{a b}^{x y} \vDash \psi\right\}^{36}$ is the mapping determined by $\psi$, suppose $\psi_{s}^{\mathcal{M}}$ is functional on $A$, i.e., that, for all $a \in A$, there is a unique $b$ such that $\psi_{s}^{\mathcal{M}}(a, b)$. Let $B=\psi_{s}^{\mathcal{M}}[A]=\left\{b: \exists a \in A, \psi_{s}^{\mathcal{M}}(a, b)\right\}$ be the range of the mapping $\psi_{s}^{\mathcal{M}}$ on $A$; we need to show that $B \in U$. Since the mapping $\psi_{s}^{\mathcal{M}}$ is functional on $A$, it must be that $|B| \leq|A|$. Hence, since $|A|<\kappa,|B|<\kappa$ so there is a cardinal $\lambda<\kappa$ such that $|B|=\lambda$. Hence, $\rho_{U}[B]=\left\{\rho_{U}(b): b \in B\right\}$ is no larger than $\lambda$ and hence, as $\kappa$ is inaccessible, $\rho_{U}[B]$ must have a strict upper bound $\alpha<\kappa$ lest it be cofinal in $\kappa$. So for all $b \in B, \rho_{U}(b)<\alpha$. Hence, by the above Fact, $B \subseteq U_{\alpha}$ and so $B \in \wp\left(U_{\alpha}\right) \subseteq U_{\alpha+1} \subseteq U$, i.e., $B \in U$.

A Model of $\mathrm{ZFCU}^{*}+$ SoA $_{\infty}$. We can transform the argument above so as to yield a model of ZFCU* by restricting the "growth" of each level to sets of size $\leq \kappa$. Accordingly, let $\wp_{\kappa}(A)=\{B \in \wp(A):|B| \leq \kappa\}$; then, for $\alpha<\kappa$ and limit ordinals $\lambda \leq \kappa$, let:

$$
\begin{aligned}
U_{0} & =A^{*} \\
U_{\alpha+1} & =U_{\alpha} \cup \wp_{\kappa}\left(U_{a}\right) \\
U_{\lambda} & =\bigcup_{\alpha<\lambda} U_{\alpha}
\end{aligned}
$$

Again we let $U=U_{\kappa}$ and we define the interpretation $\mathcal{M}=\langle U, I\rangle$ exactly as

\footnotetext{
${ }^{35}$ More specifically, regarding $\mathbf{A}_{\infty}$ : For cardinals $\lambda<\kappa$ (hence for the cardinal numbers of our model $\mathcal{M}$ ), let $A_{\lambda}^{*}$ be the set of "atoms" $\{\langle\kappa, \alpha\rangle: \alpha<\lambda\}$. We note first that, since $A_{\lambda}^{*} \subseteq$ $A^{*}=U_{0}$, it follows that $A_{\lambda}^{*} \in \wp\left(U_{0}\right) \subseteq U_{1} \subseteq U$ and hence that $A_{\lambda}^{*}$ is a set in $\mathcal{M}$. Obviously the mapping $f_{\lambda}(\alpha)=\langle\kappa, \alpha\rangle$, for $\alpha \in \lambda$, injects $\lambda$ into $A_{\lambda}^{*}$. We need only show that $f_{\lambda} \in U$. But this is clear, for $f_{\lambda}$ is the set $\{\langle\alpha,\langle\kappa, \alpha\rangle\rangle: \alpha<\lambda\} \subseteq \wp\left(\wp\left(\lambda \cup A^{*}\right)\right) \subseteq \wp\left(\wp\left(U_{\lambda}\right)\right) \subseteq U_{\lambda+2}$, so $f_{\lambda} \in \wp\left(U_{\lambda+2}\right) \subseteq U_{\lambda+3}$, so $f_{\lambda} \in U$. Regarding SoA: $A^{*} \in \wp\left(A^{*}\right)=\wp\left(U_{0}\right) \subseteq U_{1}$.

${ }^{36}$ As usual, for variables $v, s_{a}^{v}=(s \backslash\{\langle v, s(v)\rangle\}) \cup\{\langle v, a\rangle\}$.
} 
above (relative, of course, to the new definition of $U$ ). The truth of $\mathbf{A}_{\infty}$ and SoA in $\mathcal{M}$ follow more or less as in fn 35 and the truth of Rnk and all the axioms of $\mathrm{ZFCU}^{*}$ other than $\mathbf{P S}^{*}$ and $\mathbf{F}^{*}$ are unaffected by the modification in the definition of $U .{ }^{37}$ And Det is obviously true by construction, as there are no sets of size $>\kappa$ in $U$.

Regarding PS*, suppose $A \in U_{\alpha}$ and let $\wp_{<\kappa}(A)=\{B \in \wp(A):|B|<\kappa\}$ be the set of all of $A$ 's "determinable" subsets. We need to show that $\wp_{<\kappa}(A) \in$ $U$. It is easy to show by the construction of $U$ that all of $A$ 's subsets are also in $U_{\alpha}$ and, hence, that $\wp_{<\kappa}(A) \subseteq U_{\alpha}$. Hence, $\wp_{<\kappa}(A) \in \wp\left(U_{\alpha}\right) \subseteq U_{\alpha+1} \subseteq U$, so $\wp_{<\kappa}(A) \in U$, as required; so PS* is true in $\mathcal{M}$.

As for $\mathbf{F}^{*}$, suppose $\psi, s$, and $\psi_{s}^{\mathcal{M}}$ are as above and that, for a given $A \in$ $U$, either (a) $|A|<\kappa$ or, where once again $B=\psi_{s}^{\mathcal{M}}[A]$, (b) $\rho_{U}[B]$ is strictly bounded above by some $\alpha<\kappa$. In either case, it must be that $B \in U$. In the former case (a), we argue exactly as above except that, in the final sentence, we replace the powerset operator $\wp$ with $\wp_{\kappa}$. In the latter case (b), by our Fact again, every element of $B$ must be in some $U_{\beta}$, for $\beta<\alpha$, and, hence, by the cumulative nature of the "stages" $U_{\gamma}, B \subseteq U_{\alpha}$. Since $A \in U$, it follows by our construction that $|A| \leq \kappa$ so, since $\psi_{s}^{\mathcal{M}}$ is functional on $A$, it must also be that $|B| \leq\{A\}$ and hence that $|B| \leq \kappa$. So $B \in \wp_{\kappa}\left(U_{\alpha}\right) \subseteq U_{\alpha+1} \subseteq U$, i.e., $B \in U$.

\section{References}

Aken, J. V. (1986). Axioms for the Set-Theoretic Hierarchy. The Journal of Symbolic Logic, 51(4):992-1004.

Boolos, G. (1971). The Iterative Conception of Set. Journal of Philosophy, 68(8):215-231.

Cantor, G. (1932). Gesammelte Abhandlungen mathematischen und philosophischen Inhalts. Springer-Verlag.

Cantor, G. (1967). Letter to Dedekind. In van Heijenoort, J., editor, From Frege to Gödel: A Source Book in Mathematical Logic, 1879-1931, pages 113-117. Harvard University Press.

\footnotetext{
${ }^{37}$ Regarding $\mathbf{A}_{\infty}$, the reasoning is nearly identical but for the fact we need in addition to note that, for $\lambda<\kappa,\left|A_{\lambda}^{*}\right|=\lambda<\kappa$ and that $\left|f_{\lambda}\right| \leq|\lambda \times A *|=|\lambda \times \kappa|=\kappa$. We then argue similarly except that we replace every occurrence of the powerset operator $\wp$ with $\wp_{\kappa}$. Likewise regarding SoA: $\left|A^{*}\right|=\kappa$ and, hence, $A^{*} \in \wp_{\mathcal{\kappa}}\left(A^{*}\right)=\wp_{\mathcal{\kappa}}\left(U_{0}\right) \subseteq U_{1}$.
} 
Devlin, K. (1991). The Joy of Sets. New York: Springer-Verlag, 2nd edition.

Easton, W. B. (1970). Powers of Regular Cardinals. Annals of Mathematical Logic, 1(2):139-178.

Forrest, P. and Armstrong, D. M. (1984). An Argument against David Lewis' Theory of Possible Worlds. Australasian Journal of Philosophy, 62:164-168.

Fraenkel, A., Bar-Hillel, Y., and Levy, A. (1973). Foundations of set theory. page 404 .

Friedman, H. (2004). Faithful Representation in Set Theory with Atoms. http://www.cs.nyu.edu/pipermail/fom/2004-January/007845.html.

Hallett, M. (1984). Cantorian Set Theory and Limitation of Size. Oxford: Clarendon Press.

Hartogs, F. (1915). Über das Problem der Wohlordnung. Mathematische Annalen, 76:438-443.

Jané, I. (1995). The Role of the Absolutely Infinite in Cantor's Conception of Set. Erkenntnis, 42(3):375-402.

Lewis, D. (1986). On The Plurality of Worlds. New York: Oxford University Press.

Lewis, D. (1991). Parts of Classes. Oxford: Blackwell Publishers.

McGee, V. (1997). How We Learn Mathematical Language. The Philosophical Review, 106(1):35-68.

Mendelson, E. (1997). Introduction to Mathematical Logic. New York: Chapman \& Hall, 4th edition.

Menzel, C. (1984). Cantor and the Burali-Forti Paradox. The Monist, 67:92107.

Menzel, C. (1986). On the Iterative Explanation of the Paradoxes. Philosophical Studies, 49:37-61.

Moore, G. H. (1978). The Origins of Zermelo's Axiomatization of Set Theory. Journal of Philosophical Logic, 7(3):307-329. 
Moore, G. H. (1980). Beyond First-order Logic: The Historical Interplay between Mathematical Logic and Axiomatic Set Theory. History and Philosophy of Logic, 1(1-2):95-137.

Nolan, D. (1996). Recombination Unbound. Philosophical Studies, 84(2/3):239-262.

Potter, M. (2004). Set Theory and Its Philosophy. Oxford University Press.

Reinhardt, W. R. (1974). Set Existence Principles of Shoenfield, Ackermann, and Powell. Fundamenta Mathematicae, 84:5-34.

Scott, D. S. (1974). Axiomatizing Set Theory. In Jech, T. J., editor, Axiomatic Set Theory, pages 207-214. American Mathematical Society, Providence, RI.

Shoenfield, J. (1967). Mathematical Logic. Menlo Park: Addison-Wesley Publishing Co.

Sider, T. (2009). Williamson's Many Necessary Existents. Analysis, 69(21):250-258.

Uzquiano, G. (2002). Categoricity theorems and conceptions of set. Journal of philosophical logic, 31(2):181-196.

Williamson, T. (2002). Necessary Existents. In O’Hear, A., editor, Logic, Thought and Language, volume 51 of Royal Institute of Philosophy Supplement, pages 233-251. Cambridge University Press.

Zermelo, E. (1908). Untersuchungen über die Grundlagen der Mengenlehre I. Mathematische Annalen, 65:261-281.

Zermelo, E. (1930). Über grenzzahlen und mengenbereiche. Fundamenta Mathematicae, 16:29-47.

Zermelo, E. (2010). Collected Works, Volume I. New York: Springer. 